\title{
Simulations of multipactor in circular waveguides
}

\author{
V. E. Semenov, ${ }^{1}$ N. A. Zharova, ${ }^{1}$ D. Anderson, ${ }^{2}$ M. Lisak, ${ }^{2}$ and J. Puech ${ }^{3}$ \\ ${ }^{1}$ Institute of Applied Physics, Russian Academy of Sciences, 46 Ulyanov Street, \\ Nizhny Novgorod 603950, Russia \\ ${ }^{2}$ Department of Space and Geophysical Sciences, Chalmers University of Technology, \\ Gothenburg 412 95, Sweden \\ ${ }^{3}$ Centre National de la Recherche Scientifique, Toulouse 31401, France
}

(Received 23 September 2010; accepted 22 November 2010; published online 9 December 2010)

\begin{abstract}
Detailed numerical simulations have been done to investigate the properties of multipactor breakdown in circular waveguides operating in the propagating fundamental TE11 mode. Main attention has been given to a comparison between the two fundamental cases corresponding to linear and circular polarizations, respectively, of the propagating electromagnetic wave. It is found that circular polarization is considerably more susceptible to multipactor than linear polarization. The reason for this difference is clarified by a comprehensive study of the electron motion in the waveguide. (C) 2010 American Institute of Physics. [doi:10.1063/1.3526674]
\end{abstract}

\section{INTRODUCTION}

Multipactor discharges are caused by secondary electron emission when free electrons accelerated to high energies by a microwave field hit solid surfaces and are frequently observed in high power microwave components operating close to vacuum conditions. This type of discharge is an undesirable phenomenon in many practical microwave applications and the intense study of this phenomenon during the last decades has mainly been stimulated by the necessity to be able to accurately predict the multipactor threshold in different microwave subsystems. ${ }^{1-5}$ These studies have resulted in development of various numerical tools capable of simulating multipactor in different geometries including parallel plates, ${ }^{6,7}$ coaxial cables, ${ }^{8-10}$ dielectric windows, ${ }^{11-13}$ rectangular and wedge-shaped waveguides, ${ }^{14-16}$ microstrip lines, ${ }^{17}$ waveguide irises, ${ }^{18-20}$ and rf filters and transformers. ${ }^{21-23} \mathrm{~A}$ notable exception is multipactor breakdown in circular waveguides, which has been given scant interest, in spite of its technical importance. However, a relevant analysis of the problem can be found in Ref. 24 where the multipactor has been simulated numerically, although in very narrow waveguides compared to those used in practical applications. $^{25,26}$ Therefore a more detailed and general analysis is motivated for the important problem of multipactor discharges in circular waveguides. One step in this direction is presented in the present work where the multipactor discharge is simulated numerically in a waveguide having larger width than that studied in Ref. 24, in fact large enough to allow propagating TE11 modes.

The circular waveguide represents a system where the fundamental TE11 mode is degenerate with respect to polarization. This makes it possible to excite propagating waves with both linear and circular polarizations. Many practical microwave applications are based on this property and therefore it is important and interesting to analyze the dependence of the multipactor properties on the polarization of a propagating wave in a circular waveguide. Such a study has been undertaken in the present work and the main results are presented below.

\section{MODEL DESCRIPTION}

Numerical simulations of multipactor discharges in realistic circular waveguides make it necessary to consider the evolution of the multipactor during very long times containing many microwave periods. This is due to two reasons: a single transit of an electron between the walls of the waveguide takes long time (about 50 microwave periods or even more) and the establishing of the electron avalanche requires many consecutive electron transits. Previously the same problem was met in simulations of the multipactor in rectangular waveguides ${ }^{14}$ and microstrip lines. ${ }^{17}$ As emphasized in these papers, Monte Carlo simulations of the long time evolution of the multipactor discharge must be very accurate and in order to suppress statistical fluctuations it is necessary to consider a very large number of electron trajectories. The necessary number of trajectories increases rapidly with increasing duration of the simulated multipactor evolution and this makes the simulations very time consuming. The same problem also appears in simulations using the particle-in-cell (PIC) algorithm. During studies of the long time evolution, the number of computer particles typically increases or decreases significantly. As a result, the calculations become extremely slow (due to the huge number of particles) or too sensitive to stochastic fluctuations (due to too few particles). A possible solution of this problem was suggested recently in Ref. 9 where it was proposed to keep the number of computer particles within a desirable range by joining or splitting them when necessary. This approach has also been used in the present software CIRCLE which has been developed especially for multipactor simulations in circular waveguides. The software is based on the PIC algorithm and considers the motion of macroparticles (subsequently referred to as computer particles) having the same charge to mass ratio as the electrons. In principle, this algorithm makes it possible to simulate also the multipactor saturation stage when the perturbation of the microwave field by the space charge effect of 
the multipacting electrons is taken into account. However, in the present study, only the initial stage of the multipactor is considered. Consequently the microwave field is considered as undisturbed during the multipactor evolution. For simplicity only the transverse (with respect to the axis of the waveguide) motion of the particles was calculated which is justified by the small velocity of the particles compared to the speed of light.

Within the above approximation the particle motion is described by the equations (in cgs units)

$$
\ddot{x}=\eta E_{x}+\eta(\dot{y} / c) H_{z}, \quad \ddot{y}=\eta E_{y}-\eta(\dot{x} / c) H_{z},
$$

where $x$ and $y$ denote the transverse Cartesian particle coordinates, chosen so that the waveguide axis (which is directed along the $z$ axis) corresponds to the point $x=y=0$. Thus, inside the waveguide, the following inequality is fulfilled: $x^{2}+y^{2} \leq R^{2}$, where $R$ denotes the radius of the waveguide. Furthermore, a dot over the variable denotes temporal derivative, $c$ denotes the velocity of light in vacuum, $\eta$ denotes the charge to mass ratio of the computer particle which is equal to the charge to mass ratio of the electron, and $E_{x}, E_{y}$, and $H_{z}$ denote the transverse components of the electric field and the longitudinal component of the magnetic field, respectively.

In the case when a propagating TE11 mode is excited in a circular waveguide, these microwave field components can be expressed as

$$
\begin{aligned}
& E_{x}=-\sum_{j=1}^{2} A_{j}\left(\partial \Phi_{j} / \partial y\right) \cdot \cos \left(\omega t-k z+\phi_{j}\right), \\
& E_{y}=\sum_{j=1}^{2} A_{j}\left(\partial \Phi_{j} / \partial x\right) \cdot \cos \left(\omega t-k z+\phi_{j}\right), \\
& H_{z}=\sum_{j=1}^{2} A_{j}\left(k_{\perp}^{2} c / \omega\right) \Phi_{j} \cdot \sin \left(\omega t-k z+\phi_{j}\right),
\end{aligned}
$$

where $\omega$ and $k$ denote the mode frequency and propagation constant, respectively, and $A_{j}, \phi_{j}$, and $\Phi_{j}$ denote amplitude and phase and transverse mode structures of the separate waves, respectively. Specifically, using the cylindrical coordinates $r$ and $\theta$, the functions $\Phi_{j}$ can be represented as a product of Bessel and trigonometric functions,

$$
\Phi_{1}=J_{1}\left(k_{\perp} r\right) \cos \theta, \quad \Phi_{2}=J_{1}\left(k_{\perp} r\right) \sin \theta,
$$

where $k_{\perp}$ denotes the transverse wave number. When the phase shift is $\pi / 2$ (i.e., $\phi_{2}-\phi_{1}=\pi / 2$ ) and the amplitudes $A_{j}$ are equal, the microwave electric field is circularly polarized at the waveguide axis. When the phases $\phi_{j}$ are equal, any combination of the amplitudes $A_{j}$ corresponds to a linearly polarized electric field at the waveguide axis. In this case the particular choice of the amplitudes only determines the direction of the polarization. In the simulations the choice $A_{2}=0$ is made to obtain linear field polarization with $E_{x}=0$ at the waveguide axis.

The simulations are started with the launch of a number of seed electrons from a definite point at the waveguide wall. The computer seed particles have the same mass, $m$, and charge, $q$, as a single electron and have stochastic initial velocities governed by the Maxwellian probability distribution,

$$
d P=\left(\pi V_{T}^{2}\right)^{-1} \exp \left[-\left(V_{x}^{2}+V_{y}^{2}\right) / 2 V_{T}^{2}\right] d V_{x} d V_{y},
$$

where $V_{x}$ and $V_{y}$ are Cartesian components of the initial velocity in the waveguide cross section [at each point of the waveguide wall the probability given by Eq. (4) is applied only for particles moving into the waveguide] and the parameter $V_{T}$ determines the average value of the initial energy of the electrons $W_{T}=m V_{T}^{2}$. The motion of the seed electrons is governed by the undisturbed microwave field including the action of the microwave magnetic field. When the seed electrons collide with the metal wall, they release a number of secondary electrons. The emission of the secondary electrons is considered as a stochastic process with a probability distribution governed by the electron impact energy and chosen so as to correspond to Vaughan's approximation ${ }^{27}$ for the average value, $\sigma=\sigma\left(W_{\mathrm{imp}}\right)$, of the secondary emission yield,

$$
\begin{aligned}
& \sigma=0 \text { for } w<0, \\
& \sigma=\sigma_{\max } \cdot[w \exp (1-w)]^{s}, \\
& s=0.62 \text { for } 0<w<1, \quad s=0.25 \text { for } w \geq 1,
\end{aligned}
$$

where $w=\left(W_{\text {imp }}-W_{\text {min }}\right) /\left(W_{\max }-W_{\text {min }}\right), W_{\text {imp }}$ denotes the electron impact energy and the parameters $\sigma_{\max }, W_{\max }$, and $W_{\min }$ are determined by the material and the surface treatment of the wall. The secondary electrons are assumed to start with stochastic initial velocities governed by the same Maxwellian probability distribution as the seed electrons and also move under the action of the microwave field until they collide with the wall and new secondary electrons are released. This process is repeated as long as required.

When, during the simulations, the number of electrons exceeds some threshold value, $N_{\text {th }}$, the software randomly excludes one-half of the particles from further simulation and simultaneously doubles the charge and mass of the remaining particles. This operation does not disturb the motion of the remaining particles since their charge to mass ratio is not changed. In the opposite case when the number of electrons becomes less than $0.3 N_{\text {th }}$, the software splits each electron into two computer particles having half of the electron charge and mass. After this splitting, each pair of new particles is assigned the position and velocity of the original particle. Consequently the pair of new particles continues the motion of the original particle until they collide with the waveguide wall. However, the subsequent dynamics of the new particles can be different since each particle collision with the wall is treated separately using the stochastic approach. Specifically, at collisions with the waveguide wall, any computer particle (independently of its mass and charge) generates secondary particles of the same mass and charge as the primary one. The probability distribution of the secondary particles over initial velocity does not depend on the particle mass and charge and is given by the same Maxwellian distribution (4) as used for the seed electrons. The number of secondary particles is also random and the concomitant probability distribution does not depend explicitly on the 


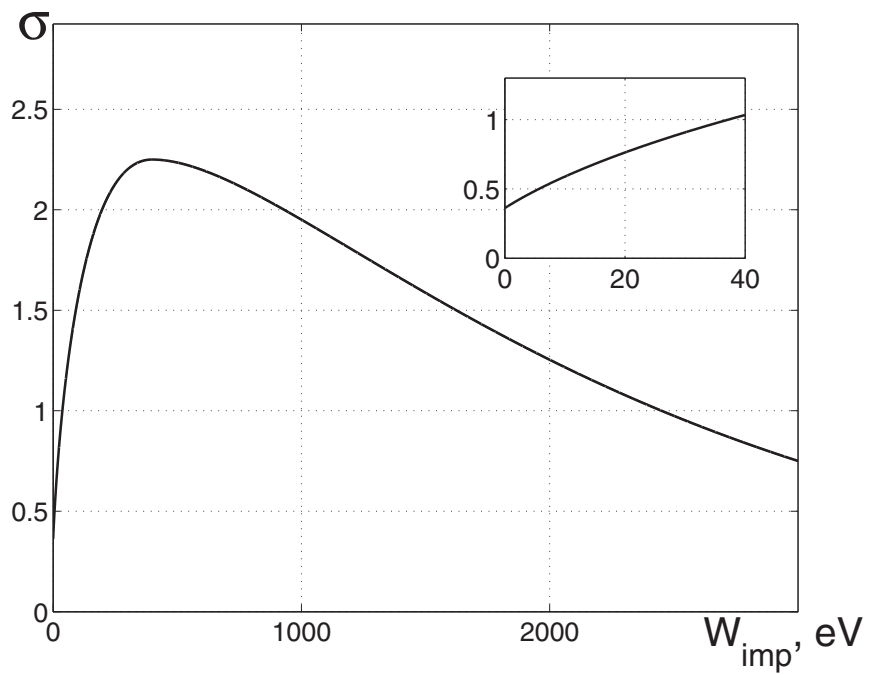

FIG. 1. Total secondary emission yield, $\sigma$, vs electron impact energy, $W_{\text {imp, }}$, as used in the simulations. The separate insert shows this dependence for small impact energy (note that $\sigma>0$ at $W_{\text {imp }} \rightarrow 0$ ).

mass and charge of the primary particle. The average number of secondary particles is given by the same approximation for the secondary emission yield (5) where the equivalent impact energy per electron is taken as $W_{\text {imp. In order to have }}$ the equivalent impact energy in all collisions, the software calculates the square of the particle impact velocity and multiplies this value by half the electron mass (independently of the actual particle mass).

The process of secondary emission results in a varying number of computer particles after each collision with the waveguide wall. However, each time when the number of computer particles exceeds $N_{\text {th }}$ or becomes less than $0.3 N_{\text {th }}$, the above procedure of particle elimination or duplication is repeated and the simulation is continued while keeping the number of computer particles within the range between $0.3 N_{\text {th }}$ and $N_{\text {th }}$. At any instant of time, information about the actual number of electrons can be obtained simply as the product of the particle factor (the ratio of the particle mass to the mass of a single electron) and the number of computer particles. As a result it becomes possible to study the evolution of the multipactor avalanche during very long times while still having both high simulation rate and high accuracy.

\section{SIMULATION RESULTS}

In a first series of simulations, the multipactor was analyzed for a case with microwave frequency, $f=10 \mathrm{GHz}$, waveguide radius, $R=1 \mathrm{~cm}$, and average initial energy of electrons, $W_{T}=2 \mathrm{eV}$. The parameters of Vaughan's approximation for the secondary emission yield (SEY) were taken to be $\sigma_{\max }=2.25, W_{\min }=-8 \mathrm{eV}$, whereas $W_{\max }$ was chosen so as to have a first cross over energy of $37.7 \mathrm{eV}$. As was shown in our previously reported simulations, ${ }^{9}$ such a combination of SEY parameters provides good agreement with experimental data for the multipactor threshold in the case of a coaxial line with copper electrodes. ${ }^{28}$ The corresponding secondary emission curve is shown in Fig. 1. Note that within

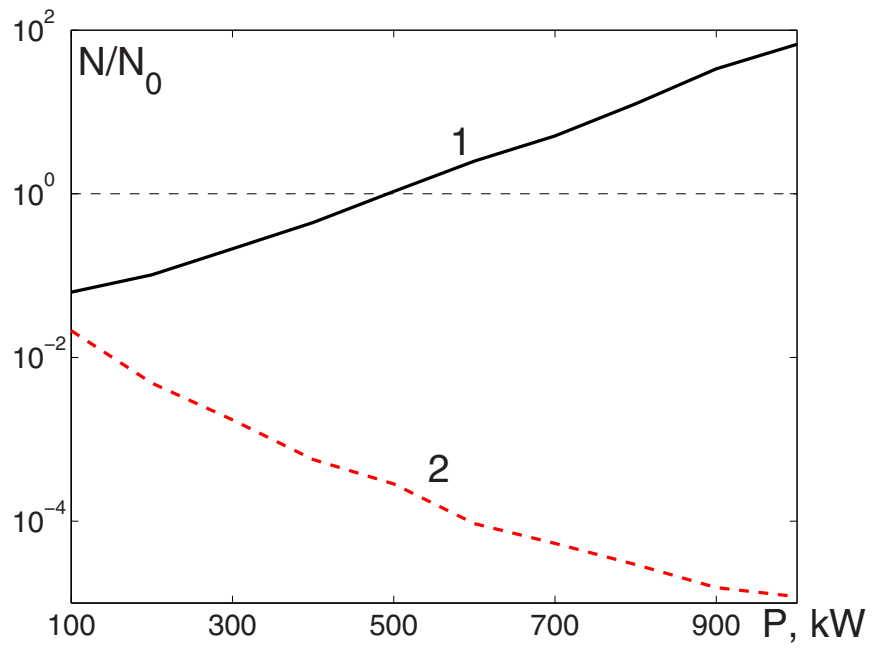

FIG. 2. (Color online) The dependence of the relative increase in electron number after 600 microwave periods on transmitted microwave power through a circular waveguide with radius $R=1 \mathrm{~cm}$ at the frequency $10 \mathrm{GHz}$. Values of $N / N_{0}$ larger than unity indicate growth of the multipactor avalanche. Curve 1 corresponds to the case of circular microwave field polarization at the waveguide axis whereas curve 2 corresponds to the case of linear microwave field polarization at the waveguide axis.

this model, the SEY does not go to zero when the electron impact energy approaches zero, which makes it possible to describe partial reflection of slow electrons from the metal surface. ${ }^{21,29-31}$ The number of seed electrons was taken to be $N_{0}=25$ 000. These electrons were launched during the first microwave period from a point with the coordinates $x=0$, $y=R$. The maximum number of computer particles was taken to be $N_{\text {th }}=50000$.

In the simulations, the primary purpose was to study how the relative increase in the average number of electrons after 600 microwave periods depended on power and polarization of the traveling electromagnetic wave transmitted through the waveguide. The simulation results are presented in Fig. 2. These results demonstrate a significant difference in the properties of the multipactor evolution for the two field polarizations. For circular polarization, the final electron number increased for increasing microwave power, as expected and similar to many other multipactor simulations in different microwave components. However, in the case of linear polarization, the opposite dependence on power was observed. In order to understand this unusual simulation result, an additional and more detailed study of the electron motion was undertaken. The corresponding results are presented in the next section.

\section{DETAILED STUDY OF ELECTRON MOTION}

A more detailed study was made of the motion of the seed electrons which depends on the emission time and the initial velocity of the electrons as well as on the power and polarization of the microwave field. In the simulations, particular attention was given to the dependence on polarization and power whereas the emission time, $t_{0}$, was kept fixed and chosen as the moment when the microwave field just begins to accelerate the electron toward the waveguide axis. Furthermore, the initial electron energy was taken to be $1 \mathrm{eV}$ 

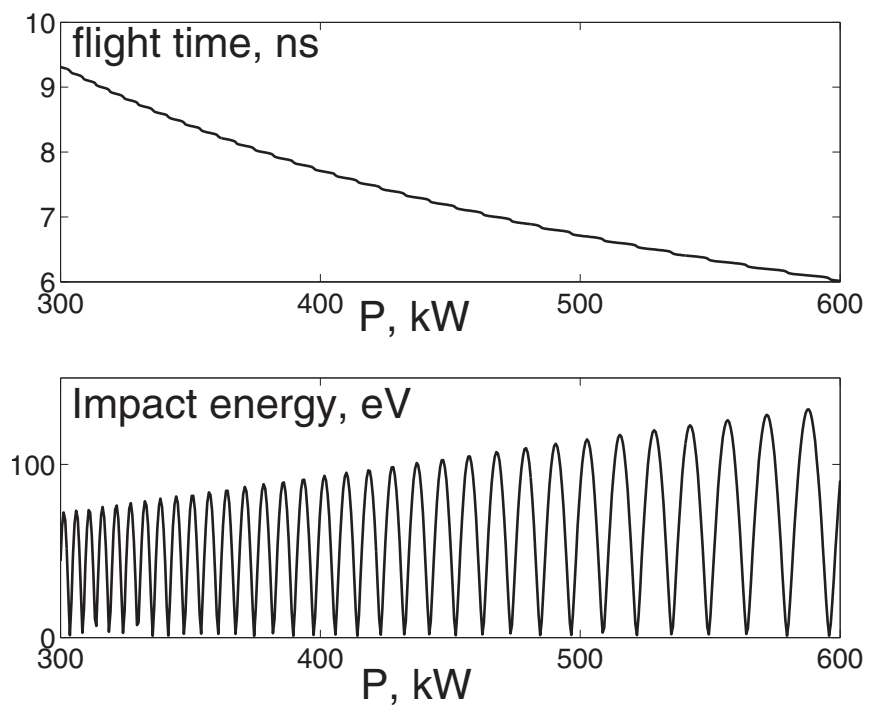

FIG. 3. The dependence of the flight time and impact energy on the microwave power for seed electrons emitted perpendicularly to the surface of the waveguide wall with initial energy $1 \mathrm{eV}$. The time of emission is chosen so that the microwave field just starts to accelerate electrons toward the waveguide axis. Case of circular field polarization at the waveguide axis.

and the electrons were assumed to start normally to the waveguide wall. Only the first trajectory of the electron crossings is considered in the simulations and no spread in initial conditions is included, which implies that no additional averaging or selection is made. The simulation results (see Figs. 3 and 4) demonstrate that the electron flight time is much greater than the microwave period and that the dependence of the impact energy on the microwave power contains many resonance peaks within each power range of $100 \mathrm{~kW}$. These results show explicitly that a smooth decrease in the final electron number with increasing power (as observed in Fig. 2 for the case of linear polarization) cannot be attributed to multipactor resonances. Moreover, the smoothness of the
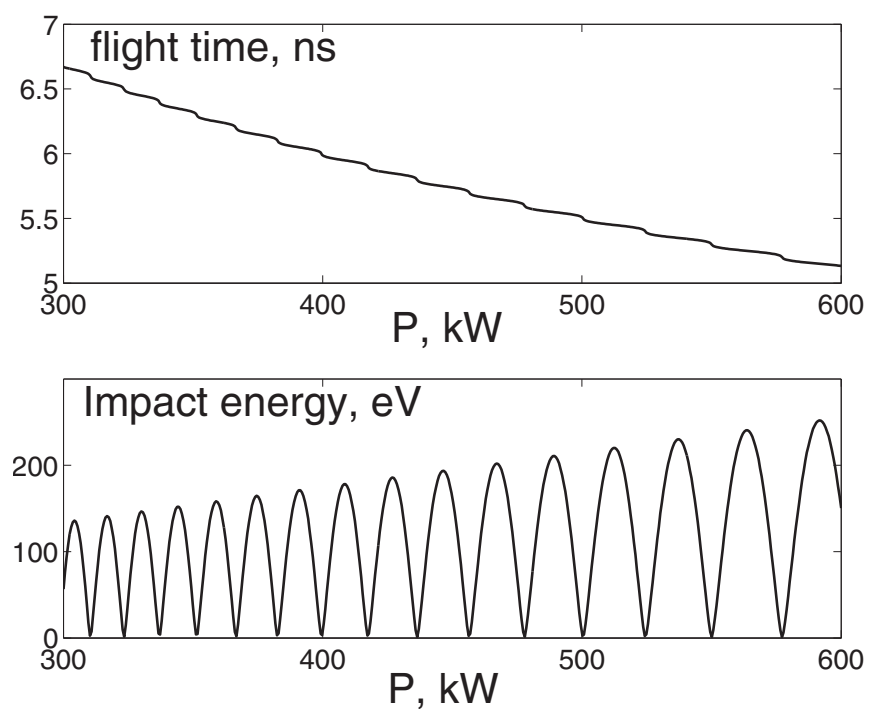

FIG. 4. The same as in Fig. 3 but for the case of linear polarization at the waveguide axis.

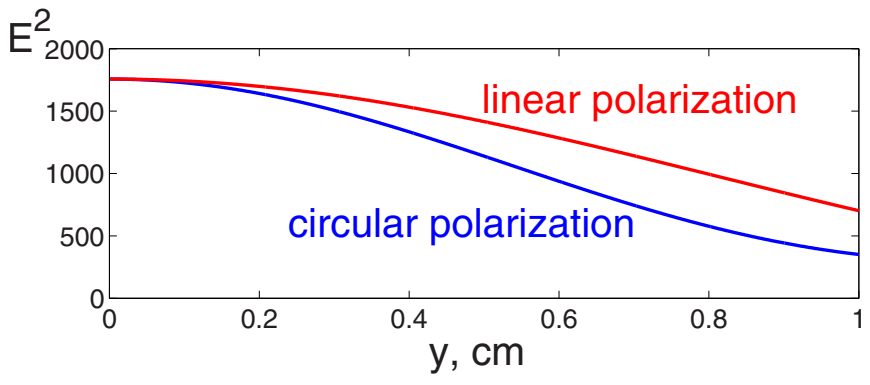

FIG. 5. (Color online) The distribution of the microwave field intensity, the square of the electric field strength (in ESU) averaged over microwave period, along the $y$ axis in the circular waveguide for two different polarizations at the same power $P=300 \mathrm{~kW}$.

curves in Fig. 2 implies that the multipactor resonances are completely suppressed by the spread of the electron initial velocity, cf. Ref. 32, for both polarizations.

It should be noted that according to Figs. 3 and 4 the impact energy increases on average with increasing power for both polarizations. The significant quantitative difference between the two polarizations is due to the higher value of the peak impact energy in the case of linear polarization and is caused simply by the higher value of the microwave electric field along the axis. However, a comparative analysis of the impact energy and the corresponding flight time reveals an important qualitative difference between linear and circular polarizations. In the case of linear polarization, each peak of impact energy is found to correspond to a flight time equal to an odd number of half microwave periods, which is characteristic for the two surface multipactor. On the other hand, in the case of circular polarization, the impact energy peaks are found to occur when the flight time equals an integer number of microwave periods, which is typical for the single surface multipactor. These results make it possible to conclude that in the case of linear polarization, the considered seed electrons cross the waveguide whereas in the case of circular polarization, the electrons return to the surface of emission. This electron behavior is caused by the repulsive action of the Miller force, ${ }^{14,33}$ which is stronger in the case of circular polarization as is clear from the distribution of the microwave field intensity along the axis shown in Fig. 5. The effect of the Miller force is illustrated in Figs. 6 and 7 where the electron motion along the $y$ axis is shown for different initial electron energies (for circular polarization) and for different times of electron emission (for linear polarization). The calculations show that in the case of linear polarization, electron reflection from the central region of the waveguide cross section is possible only for electrons which are emitted close to the microwave phase, $\phi_{0} \equiv \omega t_{0} \approx \pi / 2$. In the case of circular polarization, electrons can pass through the central region of the waveguide only when they have sufficiently high initial energy.

A tangential component of the electron initial velocity results in a deviation of the electron trajectory from the waveguide middle plane. Independently of the microwave polarization this deviation is strongly enhanced by the action of the Miller force (similarly to the case of the rectangular waveguide). ${ }^{14}$ However, the long time evolution of the elec- 


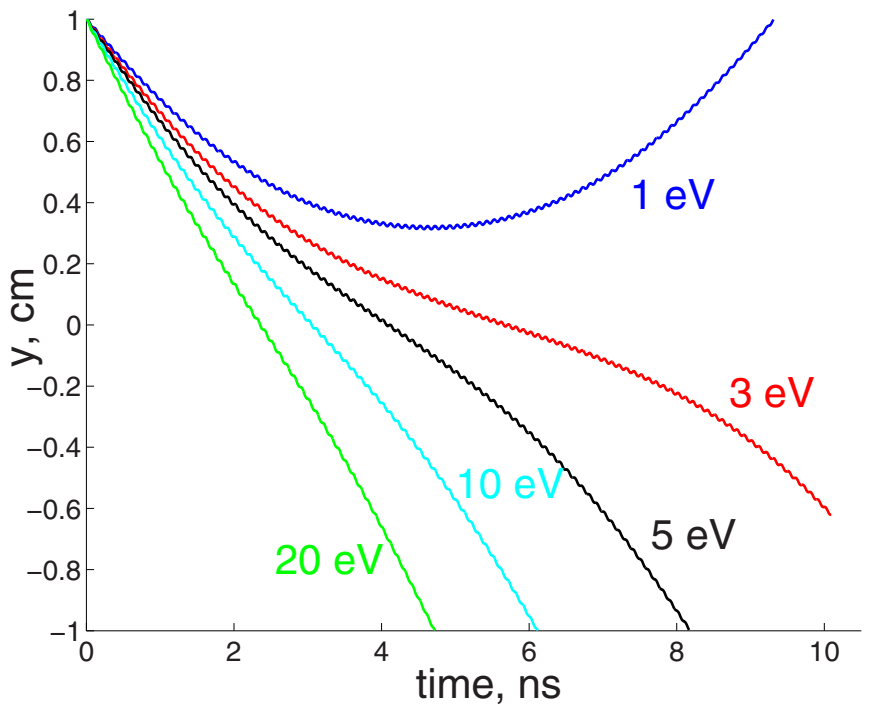

FIG. 6. (Color online) The time dependence of the $y$ coordinate of seed electrons for different electron initial energies (values indicated near each particular curve). The seed electrons are emitted perpendicularly to the waveguide wall and at zero phase of the microwave field, i.e., the electric field starts to accelerate the electrons toward the waveguide axis. Case of circular polarization with microwave power $P=300 \mathrm{~kW}$.

tron trajectories is sensitive to the field polarization. To study the long time evolution, the electron trajectories were followed also after their impact with the waveguide wall, assuming instant emission of new electrons from the point of impact. The initial velocity of each new electron was assumed to be random and to be governed by the Maxwellian probability distribution. The process was continued during 600 microwave periods using 25000 seed electrons and the spatial distribution of all electrons was averaged over the last 50 microwave periods. The result is presented in Figs. 8 and 9 which demonstrate a drastic difference between circular and linear polarizations. Actually, after 600 microwave peri-

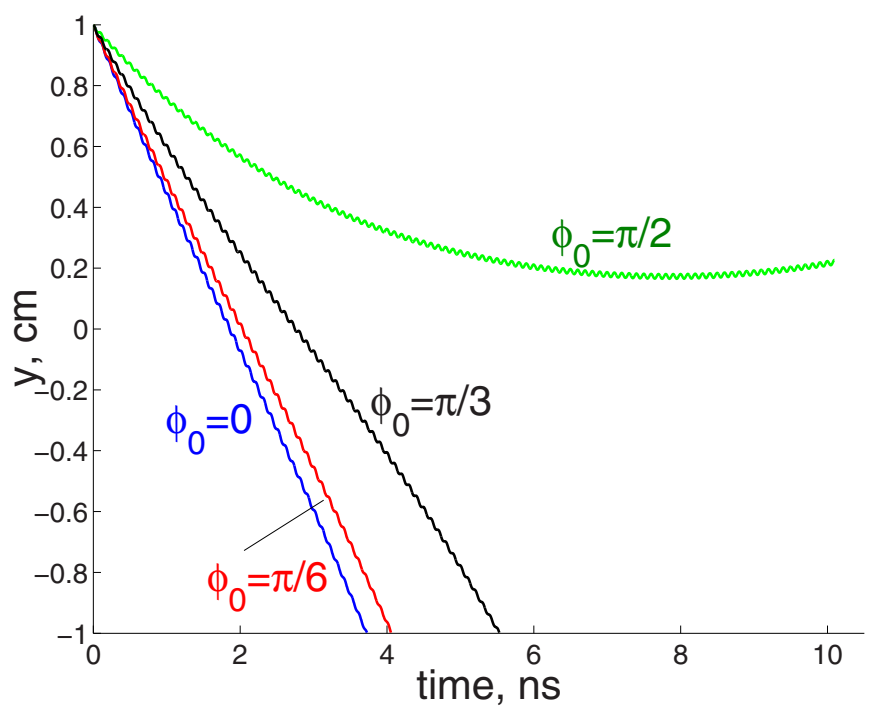

FIG. 7. (Color online) The same as in Fig. 6, but for the case of linear polarization and for different initial phases (values indicated near each particular curve) of the microwave field. The microwave power is $P=300 \mathrm{~kW}$ and a fixed initial energy of $1 \mathrm{eV}$ is used for the seed electrons.

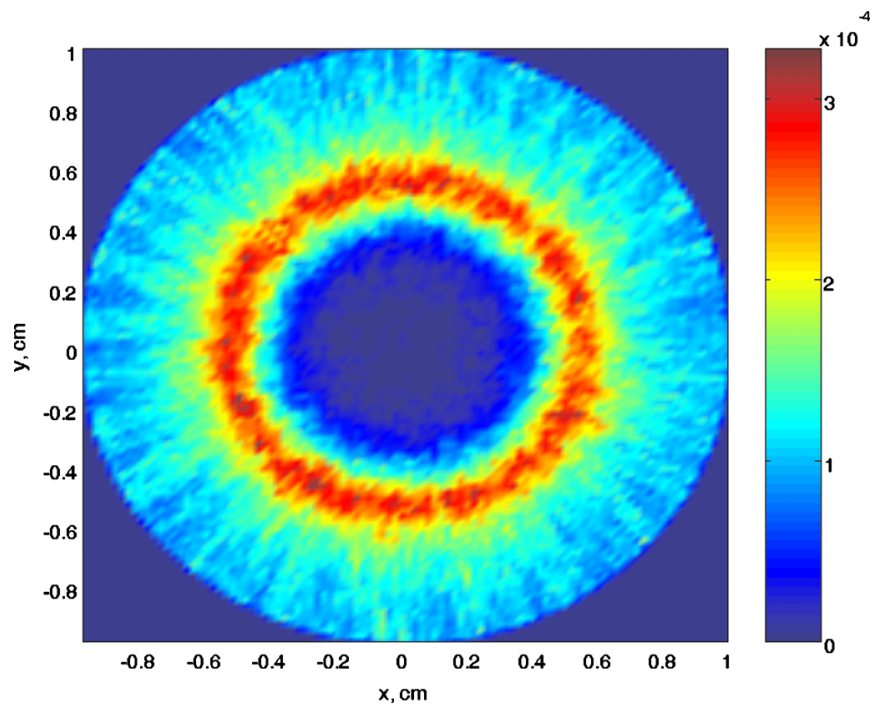

FIG. 8. (Color online) The average distribution of electrons in the waveguide cross section that is established after long time evolution in the case of circular polarization for the microwave power, $P=300 \mathrm{~kW}$. Calculations are performed for 25000 seed electrons during 600 microwave periods. Averaging is done over the last 50 periods. Average initial energy of electrons was taken to be $W_{T}=2 \mathrm{eV}$.

ods, the electrons are uniformly distributed over azimuth angle in the case of circular polarization and only the central region of the waveguide cross section looks inaccessible for the majority of the electrons. On the other hand, in the case of linear polarization, the electron distribution is quite asymmetric after 600 microwave periods. In this case the majority of electrons are pushed into the regions around the points $(x= \pm R, y=0)$ where the amplitude of the microwave electric field is close to zero. Correspondingly, the long time evolution of the electron impact energy is also quite different between the cases of circular and linear polarization (see Figs. 10 and 11). In the case of circular polarization, the average value of the electron impact energy is established at an approximately constant level after a time corresponding to

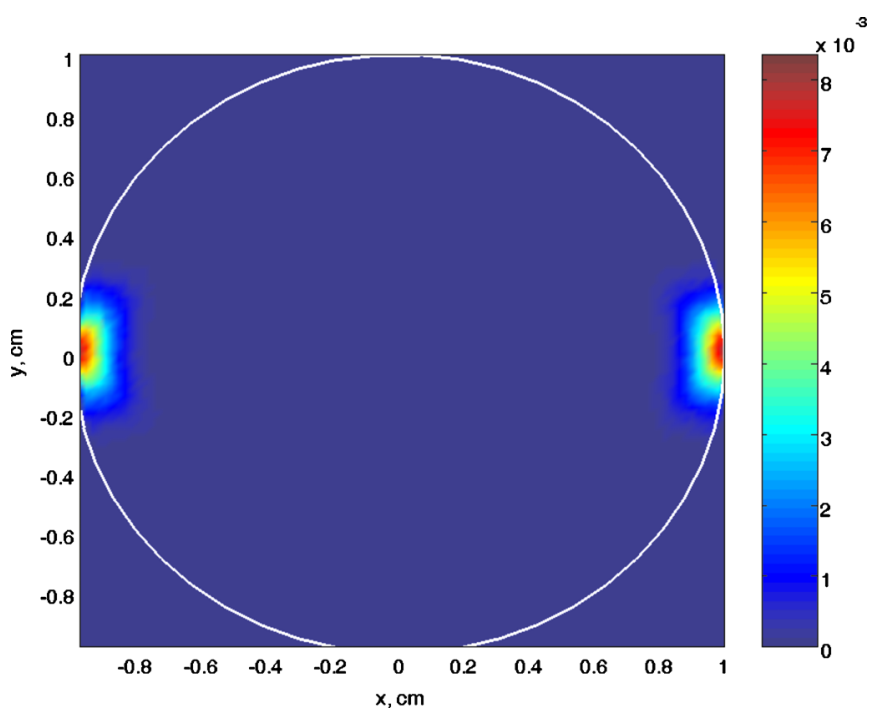

FIG. 9. (Color online) The same as in Fig. 8, but for the case of linear polarization. 


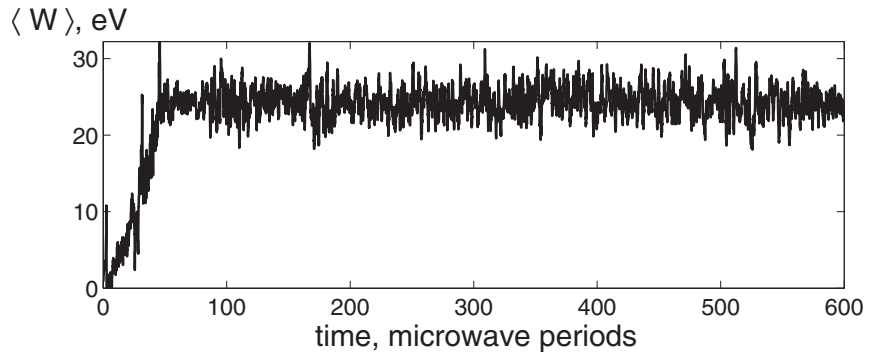

FIG. 10. The evolution of average impact energy, $\langle W\rangle$, of electrons in the case of circular polarization. Calculations are performed with the same parameters as in Fig. 8.

the first electron cross over flight between the walls. A spread of electron initial velocity results in considerable fluctuations of flight time and impact energy of the electrons. Consequently the average impact energy is considerably smaller than the peak values shown in Fig. 3. For linear field polarization, the average impact energy reaches a level very similar to that for circular polarization after the first few electron transits, but as long as electrons are pushed out of the region with strong microwave field their impact energy decreases down to a much smaller value. This particular study showed that an increase in microwave power is accompanied by a proportional increase in electron impact energy, acquired during the first few electron transits. However, in the case of linear polarization, the impact electron energy that is established after long time decreases and approaches the electron initial energy for increasing microwave power.

\section{CONCLUSION}

Numerical simulations of multipactor breakdown in circular waveguides with radii large enough to allow propagation of TE11 modes have been carried out taking into account a spread of electron initial velocity and the action of the microwave magnetic field on the electron motion. Two propagation regimes with qualitatively different polarizations of the electromagnetic wave were considered and a significant difference was found for the growth of the multipactor avalanche in the two cases. Specifically, in the case of circular polarization at the waveguide axis, an increase in the microwave power is accompanied by an increase in the electron multiplication. On the other hand, in the case of linear polarization, the higher the microwave power, the faster is the reduction in the electron number with time. To clarify the situation, a detailed study of the electron trajectories was

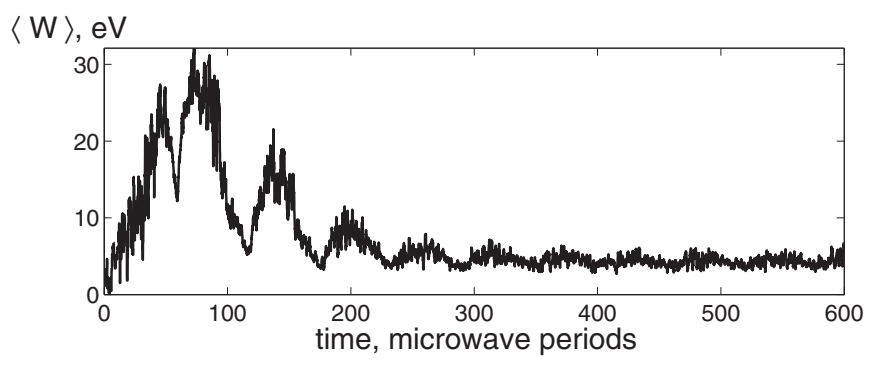

FIG. 11. The same as in Fig. 10, but for the case of linear polarization. carried out which showed that in the case of linear polarization, electrons are pushed into the region where the microwave electric field is close to zero, in accordance with the Miller force concept. The drift of the electrons out of the region with strong microwave field is equivalent to relatively strong electron losses. Although multipactor breakdown is still possible for TE11 modes with linear polarization in a circular waveguide, the simulation results indicate that multipactor growth only occurs for very high values of the secondary emission yield. It should be emphasized that these results are not in contradiction (but rather complementary) to those obtained in Ref. 24, which pertain to circular waveguides with small radii, which do not support propagating modes. The corresponding electron dynamics is then very different and corresponds to lower order multipactor resonance modes where the importance of the ponderomotive Miller force is small.

\section{ACKNOWLEDGMENTS}

This work was supported in part by the Russian Foundation for Basic Research through Grant No. 09-02-97024.

${ }^{1}$ A. Woode and J. Petit, ESA Journal 14, 467 (1990).

${ }^{2}$ A. Neuber, J. Dickens, D. Hemmert, H. Krompholz, L. L. Hatfield, and M. Kristiansen, IEEE Trans. Plasma Sci. 26, 296 (1998).

${ }^{3}$ J. G. Power, W. Gai, S. H. Gold, A. K. Kinkead, R. Konecny, C. Jing, W. Liu, and Z. Yusof, Phys. Rev. Lett. 92, 164801 (2004).

${ }^{4}$ T. Abe, T. Kageyama, K. Akai, K. Ebihara, H. Sakai, and Y. Takeuchi, Phys. Rev. ST Accel. Beams 9, 062002 (2006).

${ }^{5}$ M. Yu, IEEE Microw. Mag. 8, 88 (2007).

${ }^{6}$ A. Sazontov, M. Buyanova, V. E. Semenov, E. I. Rakova, V. Vdovicheva, D. Anderson, M. Lisak, J. Puech, and L. Lapierre, Phys. Plasmas 12, 053102 (2005).

${ }^{7}$ A. Coves, G. Torregrosa-Penalva, C. Vicente, B. Gimeno, and V. E. Boria, IEEE Trans. Electron Devices 55, 2505 (2008).

${ }^{8}$ M. Perez, C. Tienda, C. Vicente, A. Coves, G. Torregrosa, B. Gimeno, R. Barcot, V. E. Boria, and D. Raboso, IEEE MTT-S International Microwave Symposium, San Fransisco, 2006 (IEEE, New York, 2006), p. 1045.

${ }^{9}$ R. Udiljak, D. Anderson, M. Lisak, V. E. Semenov, and J. Puech, Phys. Plasmas 14, 033509 (2007).

${ }^{10}$ A. M. Perez, C. Tienda, C. Vicente, S. Anza, J. Gil, B. Gimeno, V. E. Boria, and D. Raboso, IEEE Trans. Plasma Sci. 37, 2031 (2009).

${ }^{11}$ H. C. Kim and J. P. Verboncoeur, Phys. Plasmas 12, 123504 (2005).

${ }^{12}$ M. N. Buyanova, V. E. Nechaev, and V. E. Semenov, Radiophys. Quantum Electron. 50, 893 (2007).

${ }^{13}$ A. G. Sazontov and V. E. Nechaev, Phys. Plasmas 17, 033509 (2010).

${ }^{14}$ V. E. Semenov, E. I. Rakova, D. Anderson, M. Lisak, and J. Puech, Phys. Plasmas 14, 033501 (2007).

${ }^{15}$ A. G. Sazontov, V. A. Sazontov, and N. K. Vdovicheva, Contrib. Plasma Phys. 48, 331 (2008).

${ }^{16}$ V. E. Semenov, E. I. Rakova, N. Zharova, D. Anderson, M. Lisak, and J. Puech, IEEE Trans. Plasma Sci. 36, 488 (2008).

${ }^{17}$ V. E. Semenov, E. I. Rakova, A. G. Sazontov, I. M. Nefedov, V. I. Pozdnyakova, I. A. Shereshevskii, D. Anderson, M. Lisak, and J. Puech, J. Phys. D 42, 205204 (2009).

${ }^{18}$ D. Wolk, C. Vicente, H. L. Hartnagel, M. Mattes, J. R. Mosig, and D. Raboso, Proceedings of the Fifth International Workshop on Multipactor, Corona and Passive Intermodulation in Space RF Hardware, Noordwijk, Holland, 2005, p. 93 (electronic version of the presentation available at ESTEC, Nordwijk, Holland and in CD format).

${ }^{19}$ R. Udiljak, D. Anderson, M. Lisak, J. Puech, and V. E. Semenov, IEEE Trans. Plasma Sci. 35, 388 (2007).

${ }^{20}$ V. E. Semenov, E. I. Rakova, R. Udiljak, D. Anderson, M. Lisak, and J. Puech, Phys. Plasmas 15, 033501 (2008).

${ }^{21}$ J. de Lara, F. Perez, M. Alfonseca, L. Galan, I. Montero, E. Roman, and D. Raboso, IEEE Trans. Plasma Sci. 34, 476 (2006). 
${ }^{22}$ B. Gimeno, E. Sorolla, S. Anza, C. Vicente, J. Gil, A. M. Perez, V. E. Boria, F. J. Perez-Soler, F. Quesada, A. Alvarez, and D. Raboso, Phys. Rev. E 79, 046604 (2009).

${ }^{23}$ I. Arregui, S. Anza, I. Arnedo, C. Vicente, A. Lujambio, J. Gil, M. Chudzik, B. Gimeno, T. Lopetegi, M. A. G. Laso, and V. E. Boria, Proceedings of the IEEE European Microwave Conference (EuMC), Rome, Italy 2009, p. 675.

${ }^{24}$ A. M. Perez, V. E. Boria, B. Gimeno, S. Anza, C. Vicente, and J. Gil, J. Electromagn. Waves Appl. 23, 1575 (2009).

${ }^{25}$ M. L. Riabi, R. Thabet, and M. Belmeguenai, PIER 68, 15 (2007).

${ }^{26}$ A. J. Sangster and J. Grant, Prog. Electromagn. Res. 9, 75 (2009).

${ }^{27}$ J. R. M. Vaughan, IEEE Trans. Electron Devices 36, 1963 (1989).

${ }^{28}$ R. Woo, J. Appl. Phys. 39, 1528 (1968).
${ }^{29}$ R. Cimino, I. R. Collins, M. A. Furman, M. Pivi, F. Ruggiero, G. Rumolo, and F. Zimmermann, Phys. Rev. Lett. 93, 014801 (2004).

${ }^{30}$ C. Vicente, M. Mattes, D. Wolk, B. Mottet, H. L. Hartnagel, J. R. Mosig, and D. Raboso, Proceedings of the Fifth International Workshop on Multipactor, Corona and Passive Intermodulation in Space RF Hardware, Noordwijk, Holland, 2005, p. 11 (electronic version of the presentation available at ESTEC, Nordwijk, Holland and in CD format).

${ }^{31}$ V. E. Semenov, E. I. Rakova, D. Anderson, M. Lisak, and J. Puech, IEEE Trans. Plasma Sci. 37, 1774 (2009).

${ }^{32}$ I. A. Kossyi, G. S. Lukyanchikov, V. E. Semenov, E. I. Rakova, D. Anderson, M. Lisak, and J. Puech, J. Phys. D 41, 065203 (2008).

${ }^{33}$ R. Udiljak, D. Anderson, M. Lisak, V. E. Semenov, and J. Puech, Phys. Plasmas 14, 033508 (2007). 